\title{
Article \\ Cytotoxic Effect of Graphene Oxide Nanoribbons on Escherichia coli
}

\author{
Shirong Qiang ${ }^{1,+}$, Zhengbin Li ${ }^{1,+}{ }^{,}$Li Zhang ${ }^{1}$, Dongxia Luo ${ }^{2,3, *}$, Rongyue Geng ${ }^{2}$, Xueli Zeng ${ }^{1}$, Jianjun Liang ${ }^{2}$, \\ Ping $\mathrm{Li}^{2}$ and Qiaohui Fan ${ }^{2}$
}

1 Key Laboratory of Preclinical Study for New Drugs of Gansu Province, Institute of Physiology, School of Basic Medical Sciences, Lanzhou University, Lanzhou 730000, China; qiangshirong@lzu.edu.cn (S.Q.); lizhb16@lzu.edu.cn (Z.L.); zhangl_16@lzu.edu.cn (L.Z.); zengxl17@lzu.edu.cn (X.Z.)

2 Northwest Institute of Eco-Environment and Resources, Chinese Academy of Sciences, Lanzhou 730000, China; gengrongyue18@mails.ucas.ac.cn (R.G.); liangij@lzb.ac.cn (J.L.); liping@lzb.ac.cn (P.L.); fanqiaohui@nieer.ac.cn (Q.F.)

3 College of Earth and Environmental Sciences, Lanzhou University, Lanzhou 730000, China

* Correspondence: luodx18@lzu.edu.cn; Tel.: +18-919081544

+ These authors contributed equally to this work.

Citation: Qiang, S.; Li, Z.; Zhang, L.; Luo, D.; Geng, R.; Zeng, X.; Liang, J.; Li, P.; Fan, Q. Cytotoxic Effect of Graphene Oxide Nanoribbons on Escherichia coli. Nanomaterials 2021, 11, 1339. https://doi.org/10.3390/ nano11051339

Academic Editor: Antti

Joonas Koivisto

Received: 22 April 2021

Accepted: 12 May 2021

Published: 19 May 2021

Publisher's Note: MDPI stays neutral with regard to jurisdictional claims in published maps and institutional affiliations.

Copyright: (c) 2021 by the authors. Licensee MDPI, Basel, Switzerland. This article is an open access article distributed under the terms and conditions of the Creative Commons Attribution (CC BY) license (https:// creativecommons.org/licenses/by/ $4.0 /)$.

\begin{abstract}
The biological and environmental toxicity of graphene and graphene derivatives have attracted great research interest due to their increasing applications. However, the cytotoxic mechanism is poorly understood. Here, we investigated the cytotoxic effect of graphene oxide nanoribbons (GORs) on Escherichia coli (E. coli) in an in vitro method. The fabricated GORs formed long ribbons, $200 \mathrm{~nm}$ wide. Based on the results of the MTT assay and plate-culture experiments, GORs significantly inhibited the growth and reproduction of $E$. coli in a concentration-dependent manner. We found that GORs stimulated E. coli to secrete reactive oxygen species, which then oxidized and damaged the bacterial cell membrane. Moreover, interaction between GORs and E. coli cytomembrane resulted in polysaccharide adsorption by GORs and the release of lactic dehydrogenase. Furthermore, GORs effectively depleted the metal ions as nutrients in the culture medium by adsorption. Notably, mechanical cutting by GORs was not obvious, which is quite different from the case of graphene oxide sheets to E. coli.
\end{abstract}

Keywords: graphene oxide nanoribbons; E. coli; cytotoxic effect; oxidative stress; cytomembrane damage

\section{Introduction}

Graphene is a material with a two-dimensional, monolayer, hexagonal carbon lattice structure. Due to its excellent physical, chemical, and mechanical properties, graphene and its derivatives have been extensively studied and have been used to prepare new composite materials [1-4]. Particularly, graphene oxide (GO), a graphene derivative, possesses both hydrophilic and hydrophobic oxygen-containing functional groups, such as hydroxyl, carboxyl, epoxy groups, thereby making graphene oxide soluble in water and a number of organic solvents [5]. Owing to its high solubility, GO has been widely applied in various biomedical applications, such as biological sensing, heat-based tumor targeting, drug delivery, and tissue engineering $[4,6]$.

However, because GO is continuously discharged into the atmosphere, water, and soil, this has negative effects on the environment and human beings. Therefore, increasing attention has been paid to the toxic effects of GO and its derivatives. For instance, Audira reported that GO causes behavioral dysregulation in zebrafish [7]. Zhao et al. found that GO significantly induced oxidative stress and genotoxicity in Eisenia fetida, resulting in lipid peroxidation, decreased lysosomal membrane stability, and DNA damage [8]. Furthermore, in the bacterium Azotobacter chroococcum, GO induced cell death in a dose-dependent manner owing to membrane damage and oxidative stress [9]. Due to its high dispersibility and transformation and abundant functional groups, GO inhibited the growth of freshwater 
algae through indirect toxicity via the shading effect and nutrient depletion [10]. However, in green algae, cyanobacteria and diatoms, the toxicity of GO remains various and may be species-related [11]. Hence, the mechanism of GO toxicity might be diverse according to the species of model organisms.

Model organisms are important for the evaluation of GO toxicity. Suitable model organisms would not only be beneficial to the analysis of experimental results, but also could avoid the interference caused by biological factors. Escherichia coli (E. coli) is a Gramnegative bacterium that is commonly used as a model organism in nano-toxicology studies. As a prokaryote, E. coli has a simple structure. Therefore, GO can easily break its original ecological balance through interaction with $E$. coli, which is at the bottom of the biological chain [12].

The toxicity of GO and its derivatives using an E. coli model has been investigated. For instance, reduced GO exhibited a dose-dependent and time-dependent toxic effect on E. coli [13]. GO nanosheets showed an antibacterial activity to $E$. coli by generating reactive oxygen species (ROS) [14]. In addition, Baek et al. found that GO sheet significantly damages E. coli cell membranes due to its sharp edges [15]. Among graphene, graphite oxide, GO, and reduced GO, GO showed the highest antibacterial activity against E. coli [10], and this activity difference can be attributed to surface functionality [16]. The structure of a material determines its properties. Therefore, various fabrication methods for GO might result in different toxicities and mechanisms of interaction with E. coli.

Graphene oxide nanoribbons (GORs) are a derivative of GO and are mainly fabricated by longitudinally unzipping multi-walled carbon nanotubes (CNTs) [17]. Unlike the sheet structure of GO, GORs exhibit ribbon patterns, leading to more numerous zigzag edge sites occupied by various functional groups, such as hydroxy, carboxyl, and carbonyl groups. Due to its structure, GORs are characterized by excellent mechanical strength, easy solid-liquid separation, and high specific surface area and porosity [18], thus having various practical applications.

With increasing applications, more and more GORs has been intentionally and/or unintentionally released into the environment, which might have adverse effects on the microorganisms, animals and plants, and even human beings, in the ecological environment. However, there are only limited studies on the toxic effect of GORs on microorganisms, such as E. coli, and the underlying cytotoxicity mechanism is unclear. Hence, this study fabricated GORs via the Hummers method [19], investigated GORs toxicity to E. coli, and explored the underlying mechanism of its cytotoxic effect. Our results provide a theoretical basis for the toxicity mechanism of GO and its derivatives on microorganisms and the safe application of novel nanomaterials.

\section{Materials and Methods}

\subsection{Strains and Reagents}

The E. coli ATCC 25922 strain used in this study was obtained from the School of Nuclear Science and Technology, Lanzhou University (Lanzhou, China). GORs were prepared by longitudinally unzipping multi-wall carbon nanotubes as previously described $[19,20]$. The relative medium and chemicals employed were of analytical grade and were used directly.

\subsection{Preservation and Culture of Strains}

The Luria-Bertani (LB) liquid medium was prepared by dissolving $10 \mathrm{~g}$ tryptone, $10 \mathrm{~g}$ sodium chloride, and $5 \mathrm{~g}$ yeast extract in $1 \mathrm{~L}$ Milli-Q water, adjusted to $\mathrm{pH} 7.0$, and sterilized at $120^{\circ} \mathrm{C}$ for $20 \mathrm{~min}$. To preserve the $E$. coli strain, $1 \mathrm{~mL}$ E. coli cultured overnight $\left(\mathrm{OD}_{600}=0.1\right)$ was centrifuged at $1500 \mathrm{rpm}$ for $5 \mathrm{~min}$, and the supernatant was discarded. Then, $1 \mathrm{~mL}$ phosphate-buffer saline (PBS) containing $0.3 \mathrm{~mL}$ glycerin was immitted into sediment to seal E. coli; finally, sealed E. coli was stored at $-80^{\circ} \mathrm{C}$ for subsequent use. 


\subsection{Bacteriostatic Activity}

The LB solid medium was prepared by adding $15 \mathrm{~g}$ agar powder to the LB liquid medium, adjusting $\mathrm{pH}$ to 7.5 , and sterilizing it at $120^{\circ} \mathrm{C}$ for $20 \mathrm{~min}$. Then, it was evenly applied to the Petri dish before solidification.

E. coli cultured in the LB liquid medium overnight was diluted to $\mathrm{OD}_{600}=0.1$, and fabricated GORs $(0,20$, or $200 \mu \mathrm{g} / \mathrm{mL})$ were subsequently added to diluted E. coil. The mixture was cultured with shaking (140 rpm) for $2 \mathrm{~h}$ at $37^{\circ} \mathrm{C}$ in a rotary shaker apparatus. After 1:100,000 dilution, $100 \mu \mathrm{L}$ mixture was coated with the LB solid medium and cultured in a constant-temperature incubator at $37^{\circ} \mathrm{C}$ for $24 \mathrm{~h}$. Then, the number of colonies was counted and expressed as bacterial colony formation unit (CFU).

\subsection{E. coli Survival}

The 3-(4,5-Dimethyl-2-thiazolyl)-2,5-diphenyl-2-H-tetrazolium bromide (MTT) colorimetric assay was used to evaluate cytotoxicity. MTT is a yellow dye which can be reduced to the water-insoluble and blue-purple crystal formazan by succinic dehydrogenase produced by living cells [21]. Hence, the number of living cells is associated with the color intensity of formazan, which could dissolve in dimethyl sulfoxide (DMSO).

E. coli was incubated in the LB liquid medium at $37^{\circ} \mathrm{C}$ overnight and subsequently treated with different concentrations of GORs $(0,20,50,100$, and $200 \mu \mathrm{g} / \mathrm{mL})$ for 24 , 48 , or $72 \mathrm{~h}$ in a constant-temperature incubator. E. coli was also treated with equal volumes of phosphate-buffer saline (PBS) at the same periods as control groups. After incubation, the samples were centrifuged at 10,000 rpm for $5 \mathrm{~min}$ and washed three times with PBS. Then, $200 \mu \mathrm{L}$ MTT and $600 \mu \mathrm{L}$ PBS were added to each sample, heated in a water bath at $37^{\circ} \mathrm{C}$ for $30 \mathrm{~min}$, and centrifuged at 10,000 rpm for $5 \mathrm{~min}$. After the supernatant was discarded, $3 \mathrm{~mL}$ DMSO was added, and the mixture was centrifuged again at 10,000 rpm for $5 \mathrm{~min}$. Finally, $200 \mu \mathrm{L}$ supernatant was added to a 96-well plate, and absorbance was measured at $490 \mathrm{~nm}$. Survival rate (IR; IR = experimental group/control group) was also calculated.

\subsection{Oxidative Stress}

Reactive oxygen species (ROS) produced by cells can react with unsaturated fatty acids to form lipid peroxidase, which can gradually decompose into a series of complex compounds, including malondialdehyde (MDA) [22]. Hence, the level of lipid oxidation can be indirectly determined by measuring MDA content. At high temperatures, MDA can react with thiobarbituric acid to form the reddish-brown trimethanol (3,5,5-trimethyl 2,4-dione), which has an absorption peak at $532 \mathrm{~nm}$ [23].

MDA content was determined to quantify oxidative stress. E. coli $\left(\mathrm{OD}_{600}=0.1\right)$ exposed into different concentrations $(0,20,50,100$, and $200 \mu \mathrm{g} / \mathrm{mL})$ of GORs cultured with shaking (220 rpm) for 24 or $48 \mathrm{~h}$ at $37^{\circ} \mathrm{C}$ was employed. Then, the mixture was centrifuged at $4000 \mathrm{rpm}$ for $5 \mathrm{~min}$ and washed twice with PBS buffer. The supernatant was added to $1 \mathrm{~mL}$ MDA extract, subjected to ultrasound for $10 \mathrm{~min}$ (20\% power), and centrifuged at $8000 \mathrm{rpm}\left(4^{\circ} \mathrm{C}\right)$ for $10 \mathrm{~min}$. Then, the supernatant solution $(1 \mathrm{~mL})$ was mixed with $0.5 \mathrm{M}$ hydrochloric acid $(0.3 \mathrm{~mL})$ and $1.0 \mathrm{~mL}$ TBA reagent in $0.67 \%$ and placed in a water bath at $95^{\circ} \mathrm{C}$ for $30 \mathrm{~min}$. After cooling to room temperature $\left(25 \pm 1^{\circ} \mathrm{C}\right)$, the sample was added to $4.0 \mathrm{~mL}$ methanol-butanol solution, extracted for $45 \mathrm{~min}$, and centrifuged for $10 \mathrm{~min}$ at $3000 \mathrm{rpm}$. Finally, $200 \mu \mathrm{L}$ supernatant was added to a 96-well plate, and absorbance at $535 \mathrm{~nm}$ was measured. Normal saline was used as a blank control.

$\mathrm{H}_{2} \mathrm{O}_{2}$ content is also an indicator of cellular oxidative stress. Horseradish peroxidase (HRP) is a common peroxidase with high activity and stability [24]. Briefly, $90 \mathrm{~mL}$ of E. coli after $24 \mathrm{~h}$ culture was centrifuged at $8000 \mathrm{rpm}$ for $10 \mathrm{~min}$, washed three times with $30 \mathrm{~mL}$ PBS, $2 \mathrm{~mL} 0.9 \% \mathrm{NaCl}$ was added, and homogenized manually for $10 \mathrm{~min}$. Then, the samples were centrifuged at $8000 \mathrm{rpm}\left(4^{\circ} \mathrm{C}\right)$ for $10 \mathrm{~min}$, and $200 \mu \mathrm{L}$ liquid supernatant was extracted and reacted with $20 \mu \mathrm{L}$ reaction solution (containing phenol red at $0.56 \mathrm{mmol} / \mathrm{L}$ and horseradish peroxidase at $17 \mu \mathrm{mol} / \mathrm{mL}$ ) for $2 \mathrm{~min}$. Afterwards, $10 \mu \mathrm{L} 0.5 \mathrm{M} \mathrm{NaOH}$ was 
used to terminate the reaction, and absorbance was measured at $630 \mathrm{~nm}$ using a microplate reader. Milli-Q water was used as the negative control group.

\subsection{Nutrient Depletion}

Nanomaterials can adsorb nutrients in the medium. In GORs, this nutrient depletion can be determined by measuring the change in ion concentration in the medium. Briefly, GORs at various concentrations was added into the LB liquid medium, and the samples were cultured with shaking $(220 \mathrm{rpm})$ at $37{ }^{\circ} \mathrm{C}$ for $96 \mathrm{~h}$. Then, the mixtures were filtered twice using $0.22 \mathrm{~nm}$ filter paper. The supernatant was used to culture E. coli, and E. coli was counted after $24 \mathrm{~h}$ of cultivation. The concentrations of various ions, including $\mathrm{Ca}, \mathrm{Mg}$, $\mathrm{K}, \mathrm{Fe}, \mathrm{Mn}, \mathrm{Cu}$, and $\mathrm{Zn}$, which are essential elements for $E$. coli growth, were determined using inductively coupled plasma mass spectrometry (ICP-MS). The LB liquid medium without GORs was used as the control.

\subsection{Membrane Stability and Polysaccharides Uptake}

The content of lactic dehydrogenase (LDH) could indirectly reflect the membrane stability. E. coli was exposed to GORs with serial culture system at different concentrations $(0,20,50,100$, and $200 \mu \mathrm{g} / \mathrm{mL})$ for $24 \mathrm{~h}$. After washing twice with PBS buffer, LDH level was quantified using an LDH detection kit (Solarbio) according to the manufacturer's protocol.

To investigate the affinity of GORs to the cellular surface of E. coli, we performed lipid-uptake experiments. The wavelength of polysaccharides at the maximum absorption peak was identified using a spectrophotometer. Polysaccharides at various concentrations $(100,200,300,400,500$, and $600 \mu \mathrm{g} / \mathrm{mL})$ were prepared using the standard solution, and absorbance was measured. Then, different amounts of GORs were added, and the mixtures were shaken $(180 \mathrm{rpm})$ at $37^{\circ} \mathrm{C}$ for $24 \mathrm{~h}$. Samples were centrifuged at $8000 \mathrm{rpm}$ for $10 \mathrm{~min}$, and the absorbance of the supernatant was measured.

\subsection{Characterization}

The morphologic changes in E. coli after interaction with GORs were observed by scanning electron microscopy (SEM) (Thermo, Waltham, MA, USA). Briefly, E. coli was treated with GORs at different concentrations $(0,50$, and $100 \mu \mathrm{g} / \mathrm{mL})$ for $24 \mathrm{~h}$, washed twice with PBS buffer, fixed in $2.5 \%$ glutaraldehyde for $2 \mathrm{~h}$ at $4{ }^{\circ} \mathrm{C}$, dehydrated with a series of ethanol $(30 \%, 50 \%, 70 \%, 80 \%, 90 \%$ and $100 \%)$, and permeated with tert-butanol. Finally, these samples were freeze-dried, gold-plated, and subjected to SEM.

Transmission electron microscopy (TEM) (FEI, Hillsboro, OR, USA) was also employed, and sample preparation was similar to that in SEM, except that freeze-dried samples were dissolved in Mili-Q water by ultrasound and dripped to copper mesh.

Confocal laser scan microscopy (CLSM) (Zeiss, Oberkochen, Germany) was used to observe the changes in the membrane lipid bilayer of E. coli. E. coli samples exposed to GORs $(0,20$, and $200 \mu \mathrm{g} / \mathrm{mL})$ for $24 \mathrm{~h}$ were centrifuged at 10,000 rpm for $5 \mathrm{~min}$, washed twice with PBS buffer, resuspended in $1 \mathrm{~mL}$ DiL dye in $10 \mu \mathrm{M} / \mathrm{L}$ preheated at $37^{\circ} \mathrm{C}$, and incubated at $37^{\circ} \mathrm{C}$ for $20 \mathrm{~min}$. After centrifugation at $1500 \mathrm{rpm}$ for $5 \mathrm{~min}$, the sediment was incubated with $1 \mathrm{~mL}$ LB liquid medium at $37^{\circ} \mathrm{C}$ for $5 \mathrm{~min}$ twice and centrifuged at $1500 \mathrm{rpm}$ for $5 \mathrm{~min}$. The prepared samples were developed by CLSM at an excitation wavelength of $549 \mathrm{~nm}$ and an emission wavelength of $565 \mathrm{~nm}$.

Fourier-transform infrared (FT-IR) spectroscopy (Bruker, Karlsruhe, Germany) was carried out using a Bruker Alpha instrument at 4000 to $400 \mathrm{~cm}^{-1}$. X-ray diffraction (XRD) (Rigaku, Tokyo, Japan) patterns were obtained on $X^{\prime}$ Pert Pro Panalytical instrument equipped with a rotation anode using $\mathrm{Cu}-\mathrm{K} \alpha$ radiation $(40 \mathrm{kV}$ and $30 \mathrm{~mA})$. The scanning angle started from $3^{\circ}(2 \theta)$ and continued to $80^{\circ}$ with a step interval of $0.02^{\circ}$ at a rate of $4.0^{\circ} / \mathrm{min}$. Raman spectra were measured using a JY-HR 800 micro-Raman spectrometer (Jobin yvon, Longjumeau, Frence). All samples used for FT-IR, XRD, and Raman spectroscopy were prepared after incubation, centrifugation, and freeze-drying. 


\section{Results}

\subsection{Characterization of GORs and E. coli}

In order to make sure GORs was successfully synthesized, TEM, Raman spectra, XRD and FT-IR were employed. The morphology of original GORs was observed via TEM (Figure 1a). It can be seen that the GORs were long ribbons with a width of $200 \mathrm{~nm}$, which suggested linear edges and transparent nanosheets, and were different from graphene oxide nanotubes [25]. The Raman spectra of GORs (Figure 1b) showed characteristic D and G bands at 1350 and $1570 \mathrm{~cm}^{-1}$, respectively. The XRD pattern (Figure 1c) has a sharp diffraction peak of [002] plane at $11^{\circ}$ and a broad band at $\sim 23^{\circ}$, indicating the disordered stacking of GORs. Based on the FT-IR spectra, GORs exhibited characteristic peaks at $3420 \mathrm{~cm}^{-1}$ (O-H stretching vibrations), $1730 \mathrm{~cm}^{-1}$ (C=O stretching vibrations), $1628 \mathrm{~cm}^{-1}$ ( $\mathrm{C}=\mathrm{C}$ stretching vibrations), $1232 \mathrm{~cm}^{-1}$ (C-OH stretching vibrations), $1050 \mathrm{~cm}^{-1}(\mathrm{C}-\mathrm{O}-\mathrm{C}$ stretching vibrations), and $596 \mathrm{~cm}^{-1}$ (O-H bending vibrations) [20]. According to the SEM and TEM images (Figure 1e,f), E. coli cells were short, rod-like, and $1 \mu \mathrm{m}$ in length and $0.5 \mu \mathrm{m}$ in width.
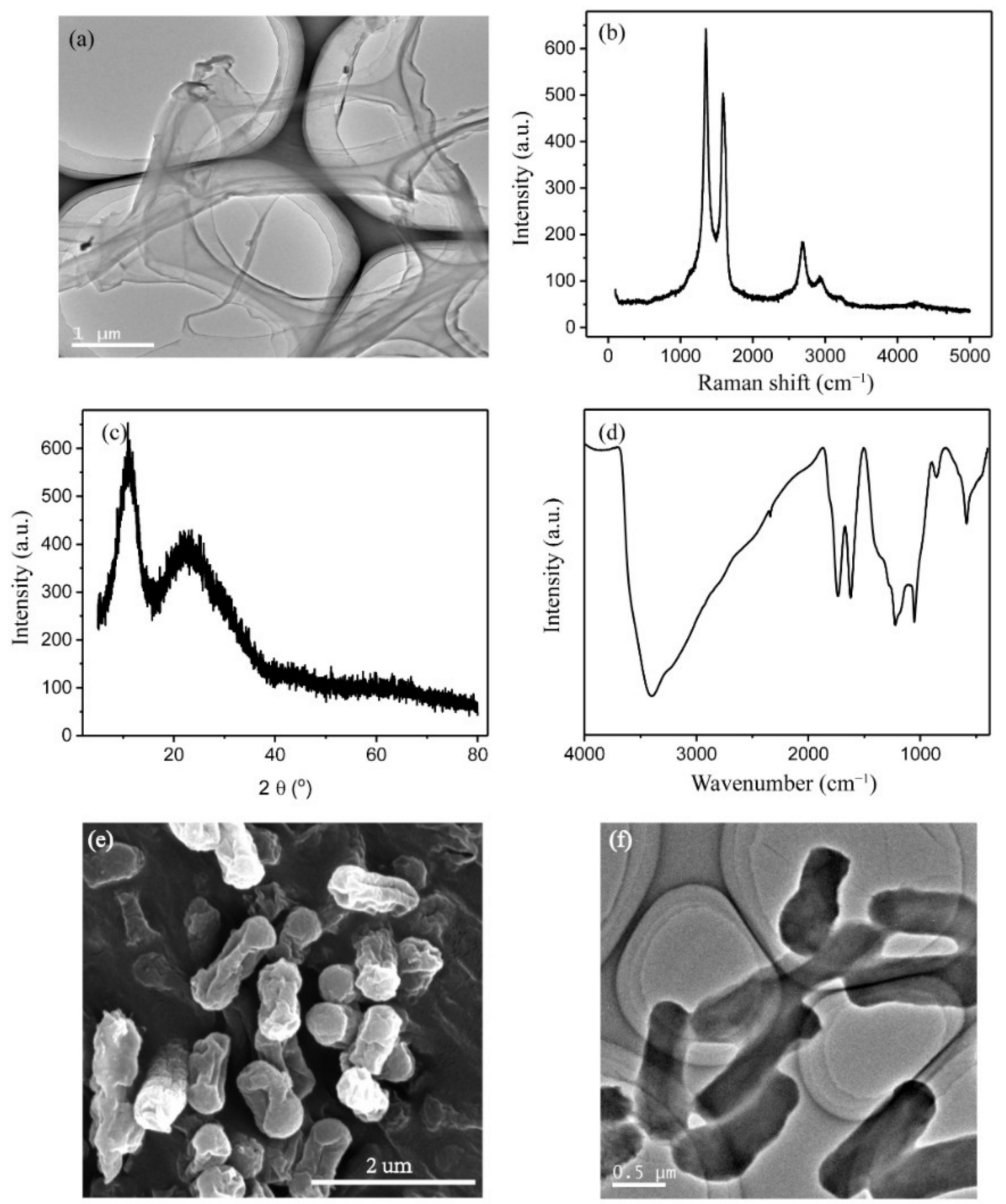

Figure 1. The TEM (a), Raman spectra (b), XRD spectra (c), and FT-IR spectra (d) of GORs, and SEM (e) and TEM (f) of E. coli.

\subsection{Toxicity Effect of GORs on E. coli}

After exposure to GORs in different concentrations, the mixture containing E. coli was coated with the LB solid medium flat and cultured for $24 \mathrm{~h}$. The dose-dependent effect of GORs treatment on E. coli growth was clearly visible to the naked eye. Particularly, fewer E. coli plaque deposits were detected following exposure to higher concentrations of GORs (Figure 2a). The number of colonies decreased with the increasing GORs concentration (Figure 2b), suggesting that GORs are considerably toxic to E. coli. This is consistent with 
the findings of Jia et al. [26], who reported that carbon nanomaterials have dose-dependent noxious effect on alveolar macrophages. In addition, the inhibition effect of GORs on $E$. coli growth was similar to that of heavy metals and was dose-dependent, further indicating that GORs have a toxicity on E. coli [27].

(a)

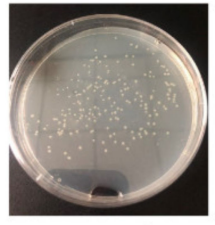

control

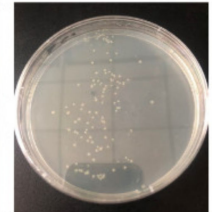

$20 \mu \mathrm{g} / \mathrm{mL}$

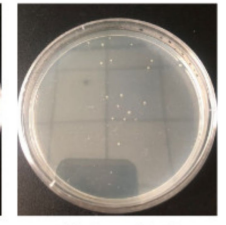

$200 \mu \mathrm{g} / \mathrm{mL}$

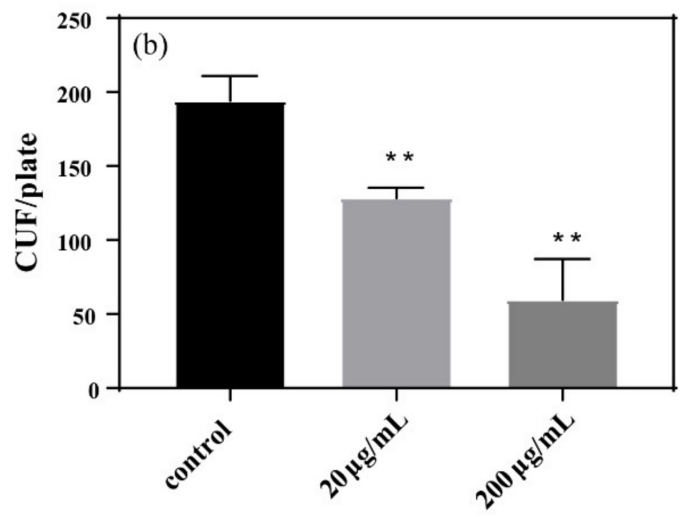

Figure 2. The result of bacteriostatic experiments after $24 \mathrm{~h}$ of culture (a) and the count of plaque of E. coli (b) (The initial E. coli solution $\mathrm{OD}_{600}$ is 0.1$)\left({ }^{* *} p<0.01\right)$.

The survival rate of E. coli also decreased with increasing GORs concentration (Figure 3), which is consistent with the results of bacteriostatic experiments. Overall, we demonstrated that GORs exert a dose-dependent toxic effect on E. coli.
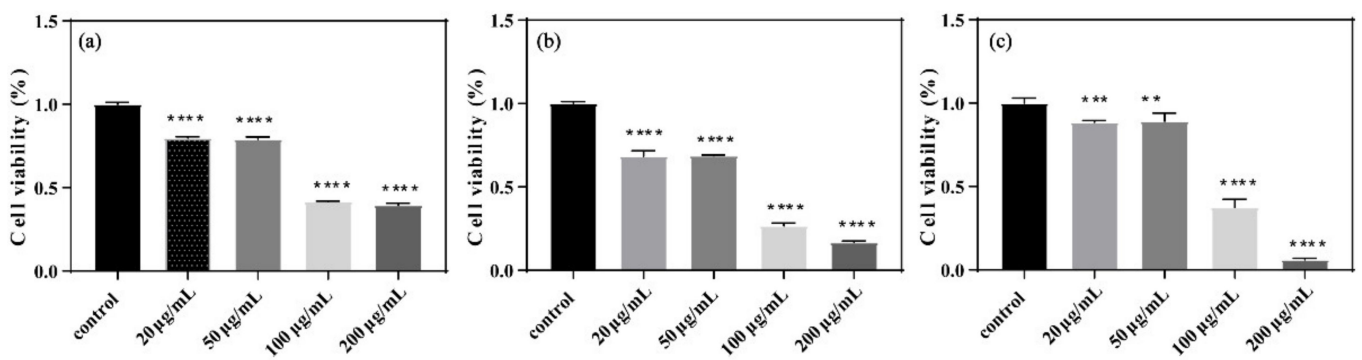

Figure 3. Survival rates of E. coli exposed to GORs in different concentrations at $24 \mathrm{~h} \mathrm{(a),} 48 \mathrm{~h} \mathrm{(b)}$, and $72 \mathrm{~h}(\mathbf{c})\left({ }^{* *} p<0.01\right.$, ${ }^{* * *} p<0.001$ and $\left.{ }^{* * * *} p<0.0001\right)$.

\subsection{Nutrient Depletion}

GORs have a large specific surface area and many adsorption sites, which are used to remove contaminants from the environment [20]. Therefore, GORs exposed to the LB liquid medium might adsorb the nutrient element, and further influence the growth and reproduction of $E$. coli. In order to solve this issue, the content of nutrient elements $(\mathrm{Fe}, \mathrm{Zn}$, $\mathrm{Ca}$, and $\mathrm{Mg}$ ) in LB medium before and after being exposed to GORs was investigated.

As shown in Figure 4a, the contents of Fe and $\mathrm{Zn}$ in the supernatant significantly decreased upon exposure to $200 \mu \mathrm{g} / \mathrm{mL}$ GORs, and those of Ca and $\mathrm{Mg}$ also decreased after interaction with GORs, suggesting that GORs can adsorb Fe, Zn, Ca and Mg. Zn is widely found in a variety of proteins and involved in processes related to bacterial 
reproduction, such as DNA and RNA synthesis [28], DNA repair [29], virus-related protein generation [30], and antibiotic resistance generation [31]. Zn is also an important component of $\mathrm{Cu}-\mathrm{Zn}$ superoxide dismutase, a metalloenzyme that plays important roles in eliminating excess reactive oxygen species [32]. Fe is an essential ingredient of RNA polymerase and RNA reductase, and is also necessary for the formation of cytochrome, hydrogenase, ferritin, and succinate dehydrogenase [33]. Thus, GORs removed the essential nutrients in the LB liquid medium via adsorption, thereby reducing their availability and inhibiting E. coli growth.
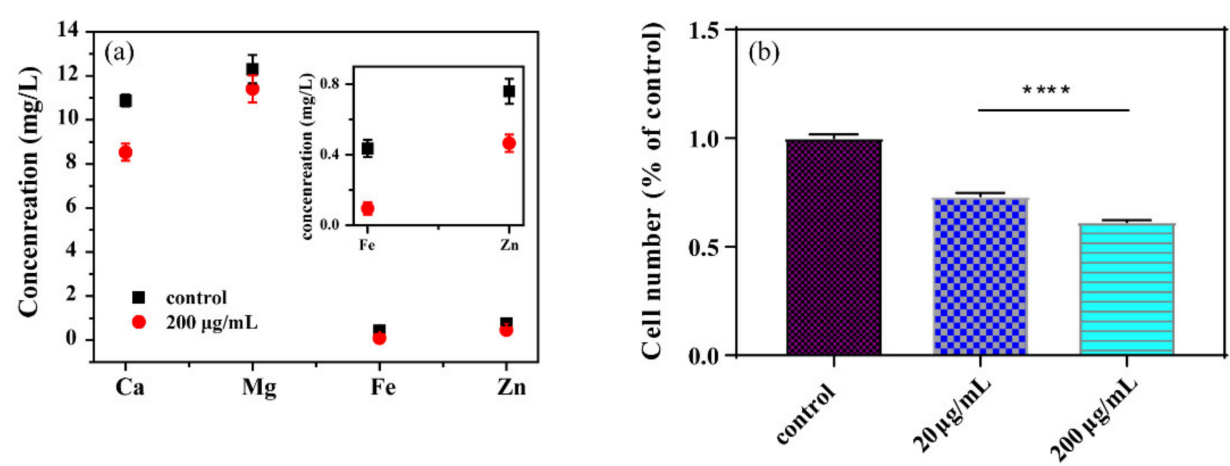

Figure 4. The content of $\mathrm{Ca}, \mathrm{Mg}$, Fe and $\mathrm{Zn}$ in supernatant of LB liquid medium after interaction with 0 and $200 \mu \mathrm{g} / \mathrm{mL}$ GORs (a), and the $E$. coli number after culture in supernatant after interaction with 0,20 and $200 \mu \mathrm{g} / \mathrm{mL}$ GORs (b) for $24 \mathrm{~h}\left({ }^{* * * *} p<0.0001\right)$.

The supernatant of LB liquid medium after being exposed to GORs was further used to culture $E$. coli for confirming the influence of nutrient element removal by GORs on E. coli reproduction. As shown in Figure 4 b, the survival rate of $E$. coli in the supernatant decreased with increasing concentrations of GORs. Thus, GORs inhibit the growth and reproduction of E. coli by competing for nutrients in the culture medium.

\subsection{Oxidative Stress}

Microorganisms generate ROS under stressful conditions [34]. Here, we used MDA and $\mathrm{H}_{2} \mathrm{O}_{2}$ as toxicological parameters to measure oxidative stress. As shown in Figure 5, MDA content in E. coli increased with exposure to increasing concentrations of GORs for $24 \mathrm{~h}$ and $48 \mathrm{~h}$. This is consistent with the observed high MDA content upon the exposure of human RPMI 8226 cells to high concentrations of GO [35]. Moreover, high levels of MDA indicate high concentrations of ROS and increased membrane oxidation, suggesting that the cytotoxic effect of GORs is closely associated with increasing ROS levels.
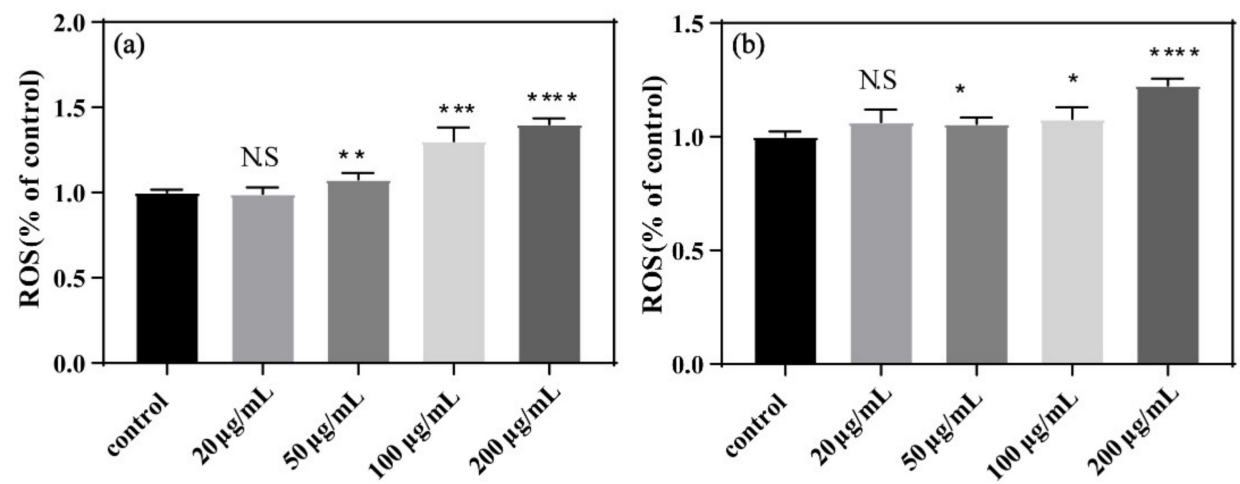

Figure 5. MDA content of E. coli exposed to GORs at different concentrations of GORs for $24 \mathrm{~h}(\mathbf{a})$ and $48 \mathrm{~h}(\mathbf{b})\left({ }^{*} p<0.05\right.$, ${ }^{* *} p<0.01,{ }^{* * *} p<0.001$ and $\left.{ }^{* * * *} p<0.0001\right)$. 
Similarly, as shown in Figure 6, $\mathrm{H}_{2} \mathrm{O}_{2}$ content produced by E. coli increased after $24 \mathrm{~h}$ exposure to GORs at increasing concentrations. This result is consistent with that of the MDA experiment, suggesting that GORs cause cytotoxic effects on E. coli via ROS generation to oxidize the bacterial cell membrane.

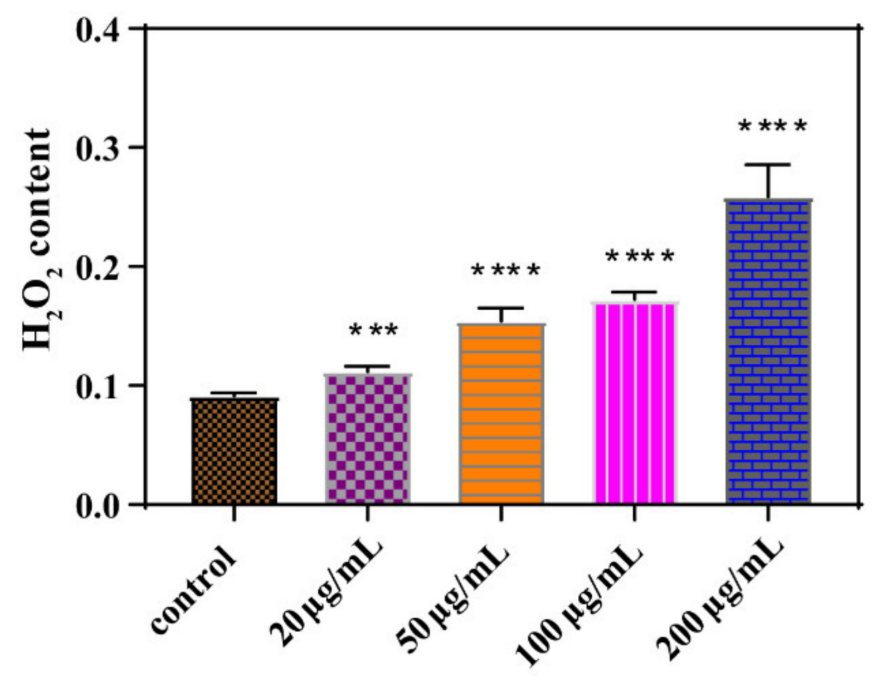

Figure 6. Peroxidase content of E. coli exposed to GORs in different concentrations ${ }^{* * *} p<0.001$, $* * * *<0.0001)$.

\subsection{Destruction of E. coli Membrane Components}

LDH is a cytoplasmic enzyme and is released into the culture medium upon cell membrane damage. Thus, we analyzed LDH levels as an indicator of membrane damage (Figure 7). LDH concentrations slightly increased with GORs concentrations in a range of $0-50 \mu \mathrm{g} / \mathrm{mL}$, suggesting that higher GORs concentrations induce more cell breakage, which led to more LDH release. However, LDH concentrations gradually decreased with GORs concentrations above $100 \mu \mathrm{g} / \mathrm{mL}$, which might be due to the excessive dose of GORs inactivated LDH causing the low detection content of LDH in the system. However, the breakage of E. coli was confirmed via a TEM image of E. coli exposed to $200 \mu \mathrm{g} / \mathrm{mL}$ GORs, which was shown in Figure 8d.

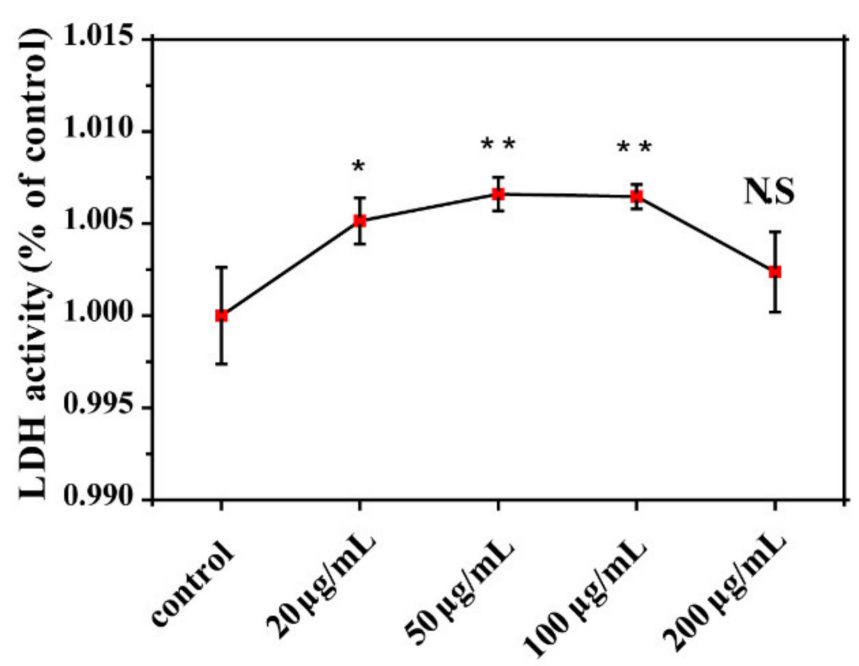

Figure 7. LDH content of $E$. coli exposed to GORs in different concentrations for $24 \mathrm{~h}(* p<0.05$, ** $p<0.01)$. 

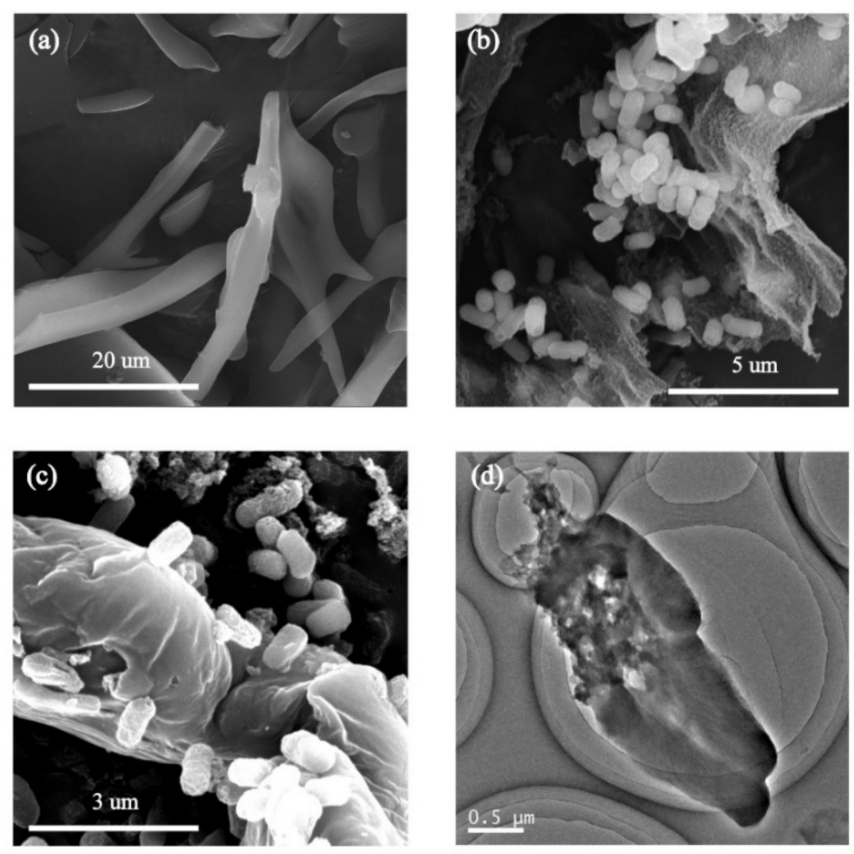

Figure 8. SEM of GORs (a) and E. coli exposed to GORs in $50 \mu \mathrm{g} / \mathrm{mL}$ (b) and $200 \mu \mathrm{g} / \mathrm{mL}$ (c), and the TEM of E. coli exposed to GORs in $200 \mu \mathrm{g} / \mathrm{mL}$ (d).

The interaction of GO and its derivatives with microorganisms is complicated. Oxidative stress of E. coli caused by GORs can oxidize the lipid components of the membrane, thereby contributing to further cell damage. Baek et al. found that GO sheet significantly damages E. coli cell membranes due to its sharp edges [1,13], suggesting the mechanical cutting may also be a factor that causes E. coli damage. As seen in the TEM and SEM images, GORs contain sharp edges (Figures 1 and 8a); however, mechanic cutting was not observed even at $200 \mu \mathrm{g} / \mathrm{mL}$ GORs concentration (Figure $8 \mathrm{~b}, \mathrm{c}$ ), suggesting that mechanical cutting effect from GORs should be very limited to E. coli in this study. Tu et al. reported that graphene nanosheets can penetrate cell membranes and extract large amounts of phospholipids due to strong dispersion interaction between graphene and lipid molecules [36]. SEM (Figure 1e) and CLSM images of E. coli (Figure 9a) showed that E. coli was evenly distributed in the control groups. However, upon treatment with GORs, E. coli aggregated around the GORs (Figure 9b-e). This was possibly due to the special affinity of GORs to E. coli membrane, resulting in the accumulation of E. coli cells around GORs. This affinity may lead to the adsorption of the membrane components by GORs, thereby increasing their bacteriostatic potency.
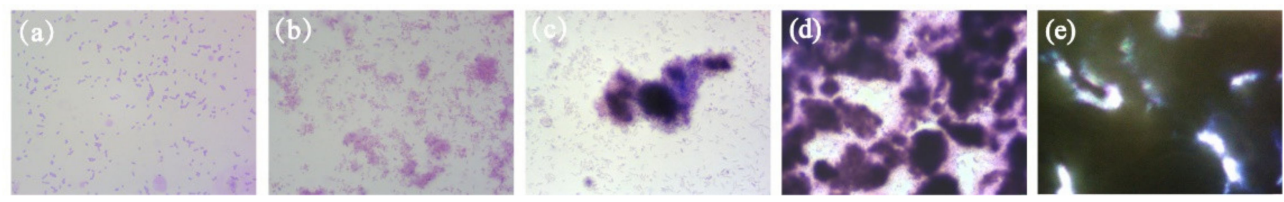

Figure 9. CLSM of E. coli exposed to GORs in $0 \mu \mathrm{g} / \mathrm{mL}$ (a), $20 \mu \mathrm{g} / \mathrm{mL}$ (b), $50 \mu \mathrm{g} / \mathrm{mL}$ (c), $100 \mu \mathrm{g} / \mathrm{mL}$ (d) and $200 \mu \mathrm{g} / \mathrm{mL}$ (e).

Polysaccharides are an abundant and important membrane component of E. coli. Thus, we investigated their interaction with GORs (Figure 10). We found that the solid phase concentration of polysaccharides at $200 \mu \mathrm{g} / \mathrm{mL}$ GORs was always higher than that at $20 \mu \mathrm{g} / \mathrm{mL}$ GORs, indicating that GORs adsorbed the polysaccharide components of the $E$. coli membrane. Therefore, the adsorption of polysaccharides may be another important mechanism for E. coli membrane damage, which caused LDH leakage. 


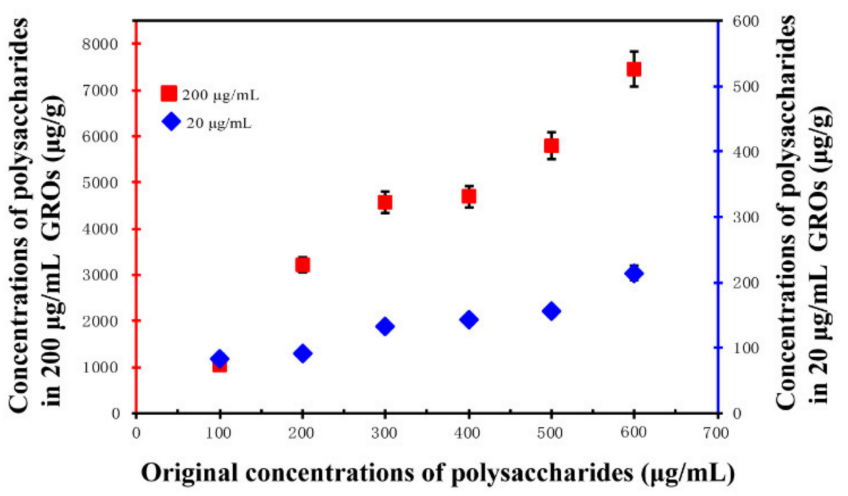

Figure 10. Changes in solid phase concentration of polysaccharides in $20 \mu \mathrm{g} / \mathrm{mL}$ and $200 \mu \mathrm{g} / \mathrm{mL}$ GORs (error bars stand for standard deviation).

\section{Conclusions}

Cytotoxic effect of GORs on E. coli and its underlying mechanism was explored in an in vitro method. GORs were long ribbons in $200 \mathrm{~nm}$ wide characterized using TEM. Plate-culture experiments showed that GORs exerted a significant cytotoxic effect on $E$. coli in a concentration-dependent manner. The depletion of nutrients in the LB medium showed that GORs adsorbed metal ions and competed with E. coli, which inhibit the growth and reproduction of E. coli. Based on increased MDA and $\mathrm{H}_{2} \mathrm{O}_{2}$ levels in the culture media, GORs stimulated E. coli to secrete ROS, which can oxidize and damage the bacterial membrane. Moreover, SEM and CLSM images revealed that E. coli aggregated around GORs, resulting in the adsorption of membrane polysaccharides by GORs to further cause membrane damage and LDH release. Notably, mechanical cutting was not obvious, which is quite different from the study between GO sheets and E. coli.

Author Contributions: Conceptualization, S.Q. and Z.L.; methodology, S.Q.; software, J.L.; validation, S.Q., Z.L. and D.L.; formal analysis, Z.L.; investigation, L.Z.; resources, S.Q.; data curation, X.Z.; writing—original draft preparation, Z.L.; writing—review and editing, D.L.; visualization, P.L.; supervision, Q.F.; project administration, R.G.; funding acquisition, S.Q. All authors have read and agreed to the published version of the manuscript.

Funding: This research was funded by National Natural Science Foundation of China (21906073 and 21876172), the "Youth Innovation Promotion Association CAS", CAS “Light of West China" Program, and Gansu Talent and Intelligence Center for Remediation of Closed and Old Deposits.

Data Availability Statement: The data presented in this study are available on request from the corresponding author.

Conflicts of Interest: The authors declare no conflict of interest.

\section{References}

1. Li, Y.; Yuan, H.; von dem Bussche, A.; Creighton, M.; Hurt, R.H.; Kane, A.B.; Gao, H. Graphene microsheets enter cells through spontaneous membrane penetration at edge asperities and corner sites. Proc. Natl. Acad. Sci. USA 2013, 110, 12295-12300. [CrossRef] [PubMed]

2. Sabyasachi, G.; Poushali, D.; Tushar, K.; Madhuparna, B.; Nikhil, K.; Amit, K.; Narayan, C. Fabrication of reduced graphene oxide/silver nanoparticles decorated conductive cotton fabric for high performing electromagnetic interference shielding and antibacterial application. Fibers Polym. 2019, 6, 1161-1171.

3. Sayan, G.; Madhuparna, B.; Tushar, K.; Subhadip, M.; Amit, K.; Narayan, C. Sonochemical green reduction to prepare Ag nanoparticles decorated graphene sheets for catalytic performance and antibacterial application. Ultrason. Sonochem. 2017, $39,577-588$.

4. Zhang, Y.; Ali, S.F.; Dervishi, E.; Xu, Y.; Li, Z.; Casciano, D.; Biris, A.S. Cytotoxicity effects of graphene and single-wall carbon nanotubes in neural phaeochromocytoma-derived PC12 cells. ACS Nano 2010, 4, 3181-3186. [CrossRef] [PubMed]

5. Qiang, S.; Wang, M.; Liang, J.; Zhao, X.; Fan, Q.; Geng, R.; Luo, D.; Li, Z.; Zhang, L. Effects of morphology regulated by Pb ${ }^{2+}$ on graphene oxide cytotoxicity: Spectroscopic and in vitro investigations. Mater. Chem. Phys. 2020, 239, 122016. [CrossRef] 
6. Seabra, A.B.; Paula, A.J.; de Lima, R.; Alves, O.L.; Duran, N. Nanotoxicity of graphene and graphene oxide. Chem. Res. Toxicol. 2014, 27, 159-168. [CrossRef] [PubMed]

7. Audira, G.; Lee, J.-S.; Siregar, P.; Malhotra, N.; Rolden, M.J.M.; Huang, J.-C.; Chen, K.H.C.; Hsu, H.-S.; Hsu, Y.; Ger, T.-R.; et al. Comparison of the chronic toxicities of graphene and graphene oxide toward adult zebrafish by using biochemical and phenomic approaches. Environ. Pollut. 2021, 278, 116907. [CrossRef]

8. Zhao, S.; Wang, Y.; Duo, L. Biochemical toxicity, lysosomal membrane stability and DNA damage induced by graphene oxide in earthworms. Environ. Pollut. 2021, 269, 116225. [CrossRef]

9. Yilihamu, A.; Ouyang, B.; Ouyang, P.; Bai, Y.; Zhang, Q.; Shi, M.; Guan, X.; Yang, S.-T. Interaction between graphene oxide and nitrogen-fixing bacterium Azotobacter chroococcum: Transformation, toxicity and nitrogen fixation. Carbon 2020, 160, 5-13. [CrossRef]

10. Zhao, J.; Cao, X.; Wang, Z.; Dai, Y.; Xing, B. Mechanistic understanding toward the toxicity of graphene-family materials to freshwater algae. Water Res. 2017, 111, 18-27. [CrossRef]

11. Yin, J.; Fan, W.; Du, J.; Feng, W.; Dong, Z.; Liu, Y.; Zhou, T. The toxicity of graphene oxide affected by algal physiological characteristics: A comparative study in cyanobacterial, green algae, diatom. Environ. Pollut. 2020, 260, 113847. [CrossRef]

12. Liu, S.; Zeng, T.H.; Hofmann, M.; Burcombe, E.; Wei, J.; Jiang, R.; Kong, J.; Chen, Y. Antibacterial activity of graphite, graphite oxide, graphene oxide, and reduced graphene oxide: Membrane and oxidative stress. ACS Nano 2011, 5, 6971-6980. [CrossRef] [PubMed]

13. Ahmad, N.S.; Abdullah, N.; Yasin, F.M. Toxicity assessment of reduced graphene oxide and titanium dioxide nanomaterials on gram-positive and gram-negative bacteria under normal laboratory lighting condition. Toxicol. Rep. 2020, 7, 693-699. [CrossRef]

14. Krishnamoorthy, K.; Umasuthan, N.; Mohan, R.; Lee, J.; Kim, S.-J. Antibacterial activity of graphene oxide nanosheets. Sci. Adv. Mater. 2012, 4, 1111-1117. [CrossRef]

15. Baek, S.; Joo, S.H.; Su, C.; Toborek, M.J. Antibacterial effects of graphene- and carbon-nanotube-based nanohybrids on Escherichia coli: Implications for treating multidrug-resistant bacteria. Environ. Manage. 2019, 247, 214-223. [CrossRef] [PubMed]

16. Xie, C.; Zhang, P.; Guo, Z.; Li, X.; Pang, Q.; Zheng, K.; He, X.; Ma, Y.; Zhang, Z.; Lynch, I. Elucidating the origin of the surface functionalization-dependent bacterial toxicity of graphene nanomaterials: Oxidative damage, physical disruption, and cell autolysis. Sci. Total Environ. 2020, 747, 141546. [CrossRef] [PubMed]

17. Wang, Y.; Hu, X.; Liu, Y.; Li, Y.; Lan, T.; Wang, C.; Liu, Y.; Yuan, D.; Cao, X.; He, H.; et al. Assembly of three-dimensional ultralight poly(amidoxime)/graphene oxide nanoribbons aerogel for efficient removal of uranium(VI) from water samples. Sci. Total Environ. 2021, 765, 142686. [CrossRef]

18. Yao, C.; Li, X.; Deng, Y.; Li, Y.; Yang, P.; Zhang, S.; Yuan, J.; Wang, R. An efficient prelithiation of graphene oxide nanoribbons wrapping silicon nanoparticles for stable $\mathrm{Li}^{+}$storage. Carbon 2020, 168, 392-403. [CrossRef]

19. Kosynkin, D.V.; Higginbotham, A.L.; Sinitskii, A.; Lomeda, J.R.; Dimiev, A.; Price, B.K.; Tour, J.M. Longitudinal unzipping of carbon nanotubes to form graphene nanoribbons. Nature 2009, 458, 872-876. [CrossRef] [PubMed]

20. Ding, Z.; Liang, J.; Zhang, W.; Wang, W.; Geng, R.; Wang, Y.; Li, P.; Fan, Q. Efficiency and active sites of the synergetic sorption and photocatalysis in $\mathrm{Cr}(\mathrm{vi})$ decontamination on a 3D oxidized graphene ribbon framework. J. Mater. Chem. A 2020, 8, 11362-11369. [CrossRef]

21. Nga, N.T.H.; Ngoc, T.T.B.; Trinh, N.T.M.; Thuoc, T.L.; Thao, D.T.P. Optimization and application of MTT assay in determining density of suspension cells. Anal. Biochem. 2020, 610, 113937. [CrossRef] [PubMed]

22. Zhang, J.; Yang, Z.; Zhang, S.; Xie, Z.; Han, S.; Wang, L.; Zhang, B.; Sun, S. Investigation of endogenous malondialdehyde through fluorescent probe MDA-6 during oxidative stress. Anal. Chim. Acta 2020, 1116, 9-15. [CrossRef] [PubMed]

23. Pirinccioglu, A.G.; Gokalp, D.; Pirinccioglu, M.; Kizil, G.; Kizil, M. Malondialdehyde (MDA) and protein carbonyl (PCO) levels as biomarkers of oxidative stress in subjects with familial hypercholesterolemia. Clin. Biochem. 2010, 43, 1220-1224. [CrossRef] [PubMed]

24. Tang, B.; Wang, Y.; Liang, H.L.; Chen, Z.Z.; He, X.W.; Shen, H.X. Studies on the oxidation reaction of tyrosine (Tyr) with $\mathrm{H}_{2} \mathrm{O}_{2}$ catalyzed by horseradish peroxidase (HRP) in alcohol-water medium by spectrofluorimetry and differential spectrophotometry. Acta Part A 2006, 63, 609-613. [CrossRef]

25. Liang, J.; Ding, Z.; Qin, H.; Li, J.; Wang, W.; Luo, D.; Geng, R.; Li, P.; Fan, Q. Ultra-fast enrichment and reduction of As(V)/Se(VI) on three dimensional graphene oxide sheets-oxidized carbon nanotubes hydrogels. Environ. Pollut. 2019, 251, 945-951. [CrossRef]

26. Jia, G.; Wang, H.F.; Yan, L.; Wang, X.; Pei, R.J.; Yan, T.; Zhao, Y.L.; Guo, X.B. Cytotoxicity of carbon nanomaterials: single-wall nanotube, multi-wall nanotube, and fullerene. Environ. Sci. Technol. 2005, 39, 1378-1383. [CrossRef] [PubMed]

27. Chen, S.H.; Ng, S.L.; Cheow, Y.L.; Ting, A.S.Y.J. A novel study based on adaptive metal tolerance behavior in fungi and SEM-EDX analysis. Hazard. Mater. 2017, 334, 132-141. [CrossRef]

28. Scrutton, M.C.; Wu, C.W.; Goldthwait, D.A. The presence and possible role of zinc in rna polymerase obtained from Escherichia coli. Proc. Natl. Acad. Sci. USA 1971, 68, 2497-2501. [CrossRef]

29. Kropachev, K.Y.; Zharkov, D.O.; Grollman, A.P. Catalytic mechanism of Escherichia coli endonuclease VIII: roles of the intercalation loop and the zinc finger. Biochemistry 2006, 45, 12039-12049. [CrossRef]

30. Ammendola, S.; Pasquali, P.; Pacello, F.; Rotilio, G.; Castor, M.; Libby, S.J.; Figueroa-Bossi, N.; Bossi, L.; Fang, F.C.; Battistoni, A. Regulatory and structural differences in the cu,zn-superoxide dismutases of salmonella enterica and their significance for virulence. J. Biol. Chem. 2008, 283, 13688-13699. [CrossRef] 
31. Meini, M.-R.; Gonzalez, L.J.; Vila, A.J. Antibiotic resistance in Zn(II)-deficient environments: Metallo- $\beta$-lactamase activation in the periplasm. Future Microbiol. 2013, 8, 947-949. [CrossRef]

32. Vignesh, K.S.; Figueroa, J.A.L.; Porollo, A.; Caruso, J.A.; Deepe, G.S., Jr. Granulocyte macrophage-colony stimulating factor induced zn sequestration enhances macrophage superoxide and limits intracellular pathogen survival. Immunity 2013, 39, 697-710.

33. Wooldridge, K.G.; Williams, P.H. Iron uptake mechanisms of pathogenic bacteria. FEMS Microbiol. Rev. 1993, 12, 325-348. [CrossRef] [PubMed]

34. Song, W.; Wang, X.; Sun, Y.; Hayat, T.; Wang, X. Bioaccumulation and transformation of U(VI) by sporangiospores of Mucor circinelloides. Chem. Eng. J. 2020, 362, 81-88. [CrossRef]

35. Wang, Y.; Wu, S.; Zhao, X.; Su, Z.; Du, L.; Sui, A. In vitro toxicity evaluation of graphene oxide on human RPMI 8226 cells. Bio Med. Mater. Eng. 2014, 24, 2007-2013. [CrossRef] [PubMed]

36. Tu, Y.; Lv, M.; Xiu, P.; Tien, H.; Zhang, M.; Castelli, M.; Liu, Z.; Huang, Q.; Fan, C.; Fang, H.; et al. Destructive extraction of phospholipids from Escherichia coli membranes by graphene nanosheets. Nat. Nanotechnol. 2013, 8, 594-601. [CrossRef] 